\title{
Análise da velocidade da bola no chute realizado por meninas praticantes de futsal
}

\author{
Analysis of ball speed in the kick made by girls practicing futsal \\ Análisis de la velocidad del balón en el patada realizado por las chicas que practican fútbol sala
}

Recebido: 02/08/2021 | Revisado: 06/08/2021 | Aceito: 07/08/2021 | Publicado: 13/08/2021

\author{
Ben Hur Soares \\ ORCID: https://orcid.org/0000-0001-6762-4799 \\ Universidade de Passo Fundo, Brasil \\ E-mail: benhur@upf.br \\ Ana Maria Zanatta \\ ORCID: https://orcid.org/0000-0001-6413-0304 \\ Universidade de Passo Fundo, Brasil \\ E-mail: ana_zanatta@hotmail.com \\ Adriano Paqualotti \\ ORCID: https://orcid.org/0000-0001-7544-9425 \\ Universidade de Passo Fundo, Brasil \\ E-mail: pasqualotti@upf.br \\ Josiane Aparecida de Jesus \\ ORCID: https://orcid.org/0000-0002-2345-2259 \\ Universidade do Oeste de Santa Catarina, Brasil \\ E-mail: josiane.jesus@unoesc.edu.br \\ Rudy José Nodari Júnior \\ ORCID: https://orcid.org/0000-0002-8375-657X \\ Salus Dermatoglifia, INOVALE, Brasil \\ Universidade do Oeste de Santa Catarina, Brasil \\ E-mail: rudy.nodarijunior@salusdermatoglifia.com.br \\ Sergio Adriano Gomes \\ ORCID: https://orcid.org/0000-0003-1989-852X \\ Universidade Católica de Brasília, Brasil \\ E-mail: congressofutsal@gmail.com \\ Mylena Aparecida Rodrigues Alves \\ ORCID: https://orcid.org/0000-0001-8614-407X \\ Universidade Federal do Paraná, Brasil \\ E-mail: mylena_cg@hotmail.com \\ Mateus Medeiros Leite \\ ORCID: https://orcid.org/0000-0002-0438-3833 \\ Universidade de Brasília, Brasil \\ E-mail: profmateusleite@gmail.com \\ Carlos Ernesto Santos Ferreira \\ ORCID: https://orcid.org/0000-0003-2397-5866 \\ Universidade Católica de Brasília, Brasil \\ E-mail: ernestobsb@gmail.com
}

\begin{abstract}
Resumo
O objetivo foi analisar a velocidade da bola na ação realizada pelo chute por meninas praticantes de futsal no contexto escolar, bem como categorizar o lado dominante e o não dominante. Foram analisadas de forma transversal e quantitativamente 53 alunas, distribuídas em suas respectivas categorias: sub $11(n=12)$, sub 13 ( $n=14)$, sub 15 (n=14) e sub $17(n=13)$, participantes nas aulas de educação física em uma escola municipal na cidade de Tapejara-RS. Para tal, cada aluna efetuou três chutes com a perna dominante, e três chutes com a perna não dominante na marcação do tiro de $10 \mathrm{~m}$ do futsal. Todas as categorias apresentaram menor velocidade média da bola no lado não dominante em relação ao lado dominante. Houve diferença significativa no lado dominante entre as categorias sub 17 e sub 11 , sendo 22,7\% maior na categoria sub 17 em relação a categoria sub $11(\mathrm{p}=0,016)$. A detecção de maior velocidade na bola após o chute, para estudantes do sexo feminino, está ligada diretamente a assimetria de utilização e estímulo dado aos segmentos nas diferentes ações diárias e específicas do futsal. Os autores sugerem um maior incentivo na participação de meninas em atividades esportivas dentro das escolinhas de futsal, pois pode elevar a maturação biológica dentro da faixa etária cronológica analisada, diminuindo o atraso no amadurecimento e na qualificação motriz de meninas que apenas fazem atividades escolares.
\end{abstract}

Palavras-chave: Futsal feminino; Chute; Análise de desempenho; Maturação sexual.

\begin{abstract}
The objective was to analyze the speed of the ball in the action performed by the kick by girls practicing futsal in the school context, as well as to categorize the dominant and non-dominant side. They were analyzed transversally and
\end{abstract}


quantitatively 53 students, distributed in their respective categories: under $11(\mathrm{n}=12)$, under $13(\mathrm{n}=14)$, under 15 $(\mathrm{n}=14)$ and under $17(\mathrm{n}=13)$, participants in the physical education classes in a municipal school in Tapejara-RS. To this end, each student made three kicks with the dominant leg, and three kicks with the non-dominant leg in the marking of the $10 \mathrm{~m}$ futsal shot. All categories showed a lower average speed of the ball on the non-dominant side in relation to the dominant side. There was a significant difference in the dominant side between the U17 and U11 categories, being $22.7 \%$ higher in the U17 category in relation to the U11 category $(\mathrm{p}=0.016)$. The detection of a higher speed on the ball after kicking, for female students, is directly linked to the asymmetry of use and stimulus given to the segments in the different daily and specific actions of futsal. The authors suggest a larger incentive in the participation of girls in sporting activities inside the futsal schools, because it can elevate the biological maturation inside of the analyzed chronological age group, diminishing the delay in the maturation and in the motor qualification of girls that only do school activities.

Keywords: Female futsal; Shooting; Performance analysis; Sexual maturation.

\section{Resumen}

El objetivo era analizar la velocidad del balón en la acción realizada por la patada de las niñas que practican fútbol sala en el contexto escolar, así como categorizar el lado dominante y no dominante. Fueron analizados transversal y cuantitativamente 53 alumnos, distribuidos en sus respectivas categorías: menores de 11 años ( $\mathrm{n}=12)$, menores de 13 años $(n=14)$, menores de 15 años $(n=14)$ y menores de 17 años $(n=13)$, participantes de las clases de educación física en una escuela municipal de Tapejara-RS. Para ello, cada alumno realizó tres patadas con la pierna dominante, y tres patadas con la pierna no dominante en el marcaje del tiro de $10 \mathrm{~m}$ de fútbol sala. Todas las categorías presentaron una menor velocidad media del balón en el lado no dominante en relación con el lado dominante. Hubo una diferencia significativa en el lado dominante entre las categorías U17 y U11, siendo un 22,7\% mayor en la categoría U17 en relación con la categoría U11 ( $\mathrm{p}=0,016)$. La detección de una mayor velocidad en el balón tras el pateo, en el caso de las alumnas, está directamente relacionada con la asimetría de uso y estímulo que reciben los segmentos en las diferentes acciones diarias y específicas del fútbol sala. Los autores sugieren un mayor incentivo en la participación de las niñas en actividades deportivas dentro de las escuelas de fútbol sala, ya que puede elevar la maduración biológica dentro del grupo de edad cronológica analizado, disminuyendo el retraso en la maduración y en la cualificación motriz de las niñas que sólo realizan actividades escolares.

Palabras clave: Fútbol femenino; Patadas; Análisis del rendimiento; Maduración sexual.

\section{Introdução}

$\mathrm{O}$ início do século $\mathrm{XX}$, a possibilidade das mulheres em praticar esportes não agradou aos olhos da população, principalmente às famílias conservadoras. Não suficiente, o Conselho Nacional Desportos regulamentou instruções proibindo as mulheres de praticarem esportes, por meio da Deliberação n. ${ }^{\circ} 7$ de 1965, em que diz: "Não é permitida a prática feminina de lutas de qualquer natureza, futebol, futebol de salão, futebol de praia, polo, halterofilismo e beisebol". Visto que diversas modalidades esportivas, entre elas o futsal e o futebol, foram considerados tradicionalmente e socialmente um ambiente masculinizado (Andreis et al., 2019).

As mulheres, para chegar ao meio esportivo, dependiam das iniciativas e subversão isoladamente. Até que na década de 80 , o esporte para o público feminino deixou de ser proibido, o que possibilitou a criação dos primeiros campeonatos às mulheres (Reis \& Arruda, 2011). Desde então, com muito esforço e quebrando muitos paradigmas, o futsal feminino vem se potencializando e profissionalizando nas questões físicas, técnicas e táticas (Slimani et al., 2016) e, mais recentemente na questão psicologia (Marenucci et al., 2020). Esse significativo avanço se deve muito ao ambiente escolar, em que, muitas das vezes, é o primeiro contato das meninas com a modalidade ou até mesmo com o esporte.

No contexto educativo das escolas, a educação física tem um papel importante para a iniciação e no envolvimento da mulher no ambiente esportivo, por considerar uma disciplina atraente para a maioria dos estudantes, é vista como uma grande fonte de motivação não só pela sua aplicabilidade como também se estende para outras disciplinas.

De acordo com Galatti e Paes (2006), a Educação Física Escolar é um componente curricular fundamental para o desenvolvimento integral do aluno por meio de vivências da cultura corporal de movimento que contribuam para o acréscimo dos domínios cognitivo, motor e afetivo, onde a prática esportiva constitui-se como um meio importante na socialização face as interações interdependentes dos agentes (professores e estudantes) envolvidos nas aulas. 
Cabe destacar que a Educação Física Escolar, como componente curricular apresenta diferentes conteúdos, modelos de ensino e abordagens pedagógicas que são estruturados em função da cultura corporal de movimento e da sociedade, onde este componente é ministrado. Nesse contexto interventivo de ensino e aprendizagem, o profissional de educação física e a escola podem ofertar atividades extras curriculares no contra turno, como os projetos esportivos funcionando como escola de iniciação esportiva. Portanto, os profissionais de educação física têm papel importante para manter os alunos ativos, proporcionando uma formação integral e o engajamento deste no ambiente escolar (Martins \& Ilha, 2020).

É visto que o futsal atrai um elevado número de praticantes, apresentando uma interdependência dos aspectos físicos, táticos, técnicos e psicológicos. No que diz respeito ao componente técnico que é expresso pelo domínio, condução, passe, cabeceio, drible e chute (Voser, 2019), estes são pré-requisitos para solucionar problemas de ordem tática oriundos da fase ofensiva do jogo (Costa et al., 2009).

Cabe destacar que o desempenho esportivo está relacionado a fatores biomecânicos, em que o chute é amplamente estudado (Barbieri et al., 2010; Nunome et al., 2002; Zanatta et al., 2020) devido a sua importância dentro de uma partida de futsal. Além disso, observa-se que há associação positiva entre a quantidade de chutes com a vitória de uma equipe em partidas oficiais de futsal (De Sousa et al, 2020; Silva et.; 2020; Rother et al., 2020; Brancher et al., 2020). Em situação específica, o padrão de movimento do chute pode ser influenciado pela situação do jogo, sendo dessa forma um fenômeno instável (Ishii et al., 2009), em que o corpo assume posturas diferentes de acordo com o alvo e velocidade da bola (Katis et al., 2013).

Considerando as aulas de educação física no ambiente formal (no contexto escolar) ou no ambiente informal (iniciação esportiva) a menina/mulher que busca e luta pelo seu espaço, encontra no futsal uma estrutura social que possibilita a inserção em modalidades esportivas coletivas dentro do ambiente escolar. Porém, há uma escassez de estudos na literatura brasileira que evidenciam a análise de características de desempenho técnico, entre eles o fundamento do chute, no sexo feminino no ambiente escolar na modalidade futsal.

Nesse contexto, o presente estudo objetivo analisar a velocidade da bola na ação realizada pelo chute por meninas praticantes de futsal no contexto escolar, bem como categorizar o lado dominante e o não dominante.

\section{Metodologia}

Trata-se de um estudo transversal de caráter quantitativo do tipo descritivo-analítico (Gil, 2002). A população deste estudo foi constituída por alunas matriculadas regularmente na Escola Municipal de Ensino Fundamental Gicondo Canali, na cidade de Tapejara-RS.

A amostra contou com 53 alunas divididas conforme sua categoria estabelecida: sub 11 (12 alunas), sub 13 (14 alunas), sub 15 (14 alunas) e na sub 17 (13 alunas). O critério de inclusão da amostra foi pela presença nas aulas de educação física em sua turma matriculada corretamente.

Todas as alunas estavam matriculadas na escola e seus responsáveis assinaram um Termo de Consentimento Livre e Esclarecido e de assentimento, conforme Resolução 466/2012 do Conselho Nacional de Saúde e de acordo com o preconizado pela Declaração de Helsinki. Esta pesquisa obteve a aprovação pelo Comitê de Ética em Pesquisa em Seres Humanos, da Fundação Universidade de Passo Fundo de acordo com os padrões éticos de normas e diretrizes regulamentadoras da pesquisa envolvendo seres humanos, sob o Parecer $\mathrm{n}^{\circ}$ 2.929.785.

O instrumento utilizado para a coleta de dados foi um Radar, medidor de velocidade da marca MUNI QUIP, modelo QUIP/KGP, com o objetivo de analisar a velocidade da bola nos chutes em direção ao gol, o mesmo foi colocado atrás da meta.

A coleta dos dados referente a velocidade da bola realizado por meio de um chute ocorreu no ginásio da Escola Giocondo Canali, localizado na Avenida Sete de Setembro - Nr 2225, Bairro São Paulo, localizado na cidade de Tapejara-RS. 
Utilizou-se bolas da marca PENALTY, específicas por categorias, sendo utilizada a bola MAX 200, com peso entre 350 a 380 gramas para as categorias sub-11 e sub-13, e, a bola MAX 1000, com peso entre 410 a 440 gramas, para as categorias sub-15 e 17.

Os dados foram coletados e preenchidos in loco durante um turno do dia, com hora pré-agendada. Antes de efetuar o teste foi realizado um aquecimento padronizado com duração de $10 \mathrm{~min}$, com movimentos básicos de deslocamentos, aç̃̃es técnicas de domínio e passe, em diferentes distâncias e alongamentos com ênfase nos membros inferiores.

A amostra efetuou três chutes com a perna dominante e três chutes com a perna não dominante, anteriormente indicados para o avaliador. Os chutes foram desferidos a partir do tiro de $10 \mathrm{~m}$ (segundo tiro penal) da quadra conforme determinam as regras oficiais da modalidade. Dentre as cobranças realizadas, foi selecionado o chute de maior velocidade desferido por cada um dos lados determinados.

\section{Análise estatística}

Todas as análises foram realizadas usando o Statistical Package for the Social Sciences (IBM SPSS, versão 25.0). O teste de Shapiro-Wilk foi utilizado para verificar a distribuição dos dados. A análise descritiva foi utilizada para apresentar os dados em média e desvio padrão. ANOVA fatorial de delineamento misto $(4 \times 2)$ foi utilizada com a finalidade de comparar a velocidade da bola nas diferentes categorias (Sub11 X Sub13 X Sub15 X Sub17) e nas dominâncias (dominante x não dominante). Tratamento de Bonferroni foi escolhido para identificar as diferenças significativas. Adotou-se o p-value de 5\% $(\mathrm{p} \leq 0,05)$ como nível de significância.

\section{Resultados}

Participaram do presente estudo 53 atletas divididas por categorias, sub $11(\mathrm{n}=12)$, sub $13(\mathrm{n}=14)$, sub 15 ( $\mathrm{n}=14)$ e sub $17(\mathrm{n}=13)$. Todas as categorias apresentaram menor velocidade média da bola no lado não dominante em relação ao lado dominante. Na categoria sub 11 , a diferença da velocidade média foi de $-12,2 \%$ menor no lado não dominante $(\mathrm{p}=0,031)$, 22,3\% na categoria sub 13 ( $p<0,001),-16,3 \%$ na categoria sub $15(\mathrm{p}<0,001)$ e $-13,3 \%$ na categoria sub $17(\mathrm{p}=0,003)$ como demonstrado na tabela 1.

Apenas para o lado dominante foram observadas diferenças entre as categorias, sendo esta apenas entre a categoria sub 17 e sub 11, sendo 22,7\% maior na categoria sub 17 em relação a categoria sub 11 ( $\mathrm{p}=0,016$ ). Para o lado não dominante, não foram observadas nenhuma diferença significativa entre as categorias ( $p>0,05)$, conforme demonstrado na Tabela 1.

Tabela 1. Comparação da velocidade da bola após o chute, entre o lado dominante e não dominante para cada categoria.

\begin{tabular}{lccc}
\hline & \multicolumn{3}{c}{ Velocidade média da bola em Km/h } \\
\hline & Dominante & Não Dominante & $\Delta$ \% dif \\
\cline { 2 - 4 } Sub 11 $(\mathbf{n = 1 2})$ & $41,0 \pm 5,5$ & $36,0 \pm 8,2^{*}$ & $-12,2 \%$ \\
Sub 13 $(\mathbf{n = 1 4})$ & $46,8 \pm 9,4$ & $36,4 \pm 8,1^{*}$ & $-22,3 \%$ \\
Sub 15 $(\mathbf{n = 1 4})$ & $48,6 \pm 9,2$ & $40,6 \pm 8,7^{*}$ & $-16,3 \%$ \\
Sub 17 $(\mathbf{n = 1 3})$ & $50,3 \pm 1,8^{\dagger}$ & $43,6 \pm 2,6^{*}$ & $-13,3 \%$ \\
Total $(\mathbf{n = 5 3})$ & $46,8 \pm 7,9$ & $39,2 \pm 7,8$ & $-16,2 \%$ \\
\hline
\end{tabular}

Notas: os dados são apresentados em média \pm desvio padrão e diferente percentual. * Diferença significativa intragrupo em relação ao membro dominante $(\mathrm{p} \leq 0,05)$. キ Diferença significativa em relação a categoria sub $11(\mathrm{p} \leq 0,05)$. Fonte: Autores.

Na Figura 1 está apresentada de forma gráfica a linearidade da velocidade da bola nas diferentes categorias nos lados dominantes e não dominantes. 
Figura 1. Demonstrativo da linearidade das médias obtidas nos chutes com o membro dominante e não dominante nas diferentes categorias avaliadas.

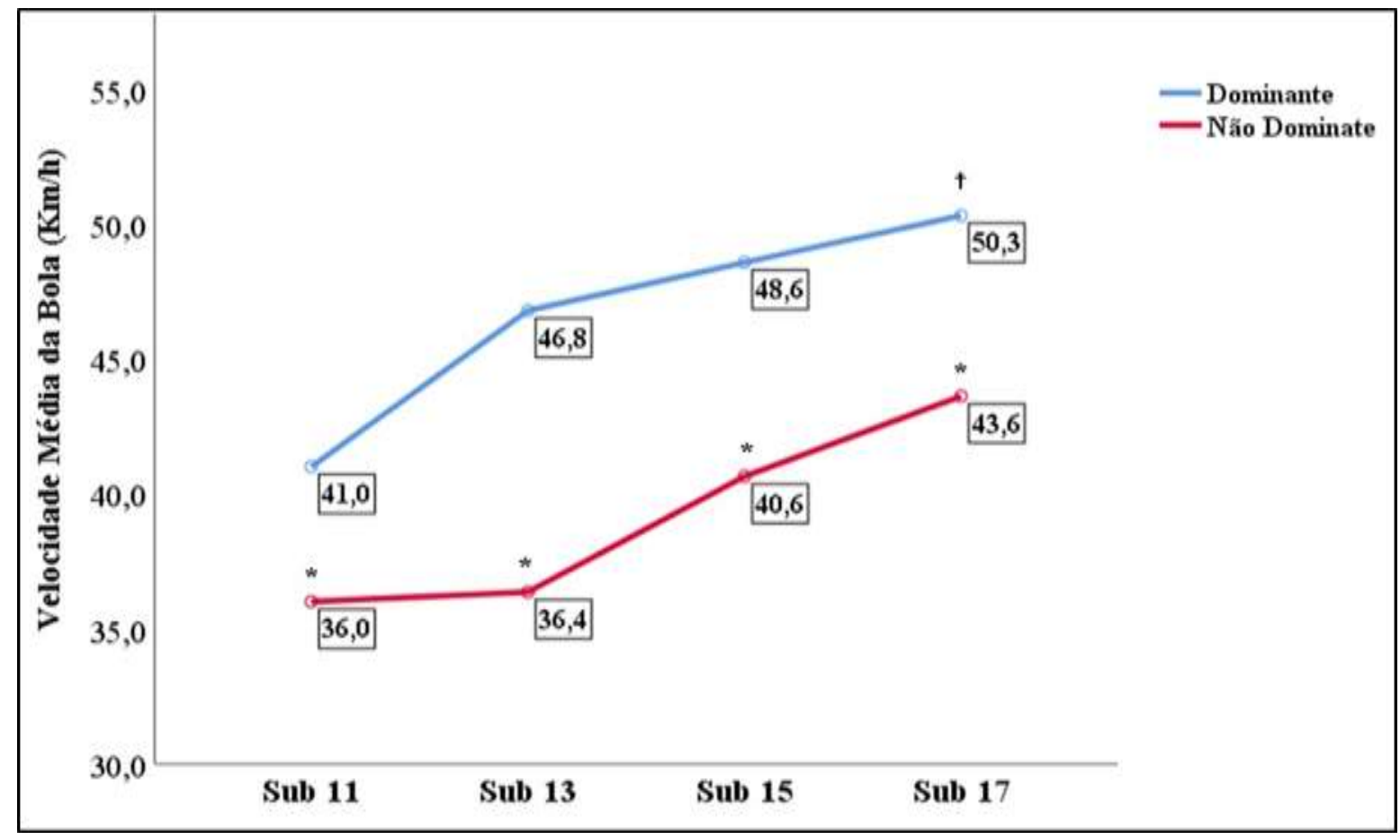

Fonte: Autores (2021).

\section{Discussão}

O objetivo da presente pesquisa foi analisar a velocidade da bola na ação realizada pelo chute por meninas praticantes de futsal no contexto escolar. O chute, entre os diversos fundamentos do futsal, é considerado o mais prestigiado, pois, em sua grande parte, antecedo o gol (Barbieri et al., 2008). Portanto, este tem sido alvo de diversos estudos (Teixeira, 2004; Nunome et al., 2006; Santiago et al., 2007).

É possível perceber, que em todas as categorias a velocidade do chute do lado dominante foi superior ao lado não dominante, demonstrando que ao longo das diferentes faixas etárias, o desenvolvimento dos componentes físicos como força, velocidade e técnica de chutar a bola, entre ambas as segmentações, não são simétricos, demonstrando uma preferência pelo uso do lado dominante.

A relevância a assimetria, foi retratada por Barbieri (2008), encontrando também, uma maior velocidade do chute no membro dominante. Esta caracterização de preferência, gera um número mais elevado de estímulos para o lado dominante, sendo que, quando voltada para o meio esportivo, esta pode ser mais crônica, constatações retratadas também por Haywood e Getchell (2004).

Quando se compara com avaliação da velocidade do chute na categoria Sub 11 no naipe masculino, verificou-se uma velocidade maior no chute desferido pelos meninos (Endres et al., 2003), por outro lado, ao comparar com os achados de Zanatta et al. (2020), que avaliaram meninas praticantes de futsal em escolinha, a diferença da velocidade nos membros dominante e não dominante foram superior pró praticantes, determinando que os estímulos mais sistemáticos elevam esta capacidade, apresentando uma velocidade maior de $17,1 \%$ no lado dominante e $19,4 \%$ no lado não dominante.

$\mathrm{Na}$ categoria Sub 13, os achados se repetem, onde as meninas que fizeram parte deste estudo, apresentam também um déficit de velocidade, quando comparado aos meninos (Endres et al., 2003), e ao comparar com meninas que praticam 
treinamentos específicos de futsal (Zanatta et al., 2020), os achados foram maiores nesta categoria, sendo 21,3\% no lado dominante e $38,9 \%$ no não dominante.

Nas demais categorias avaliadas (Sub 15 e 17), a diferença a favor dos meninos se repete (Endres et al, 2003), bem como para o grupo de meninas que participam de escolinha (Zanatta et al., 2020), sendo de 30,6\% no lado dominante e 25,6\% no lado não dominante na categoria Sub 15, e na Sub 17 a diferença é ainda maior, 40\% no lado dominante e 29,5\% no não dominante.

Os tipos de chutes variam de acordo com o objetivo de sua execução, a posição e forma que a bola se encontra, a posição do corpo, a parte do pé que toca a bola, entre outros (Santiago, 2006). Porém quando ele é realizado com máxima velocidade, ocorre prejuízo na precisão (Barbieri, 2005).

Para Barbieri, Lima Júnior e Gobbi (2006), as diferenças entre os chutes realizados com membro dominante e não dominante, podem sofrer interferências das seguintes variáveis: posicionamento do pé de suporte em relação a bola, largura do penúltimo e último passo da corrida de aproximação, amplitude do último passo da corrida de aproximação e velocidade da bola. Indicando, portanto, a existência de assimetria entre membros pode estar diretamente ligada também a esses fatores.

A relação apresentada aqui no estudo, que compara meninas que participam apenas das atividades físicas escolares e praticantes de escolinha corroboram com as relações positivas entre a prática regular e orientada do exercício físico com melhoria nos níveis de aptidão física e saúde nas mais diferentes faixas etárias (Matsudo et al., 2015).

Quando avaliamos modalidades esportivas, é comum categorizar através das faixas etárias, porém, é comum a existência de inúmeros fatores que podem influenciar nos resultados (Malina, 2014; Rebelo et al., 2013). Merece destaque a maturação sexual (Godoi Filho \& Farias, 2015; Freitas et al., 2015) que apresenta diferentes estágios na mesma idade cronológica, podendo por vezes interferir diretamente nos resultados coletados (Guedes, 2011).

Mesmo que a presente pesquisa não tenha mensurado o desenvolvimento maturacional, cabe ressaltar que os níveis de testosterona na fase púbere dos meninos apresentam um pico (Culbert et al., 2014), elevando sua massa corporal e consequentemente sua força, fato que explica as diferenças encontradas entre as velocidades da bola e os sexos. As diferenças de hormônios sexuais entre meninos e meninas, resultam em respostas de performance física, principalmente na força (Santos et al., 2011).

\section{Conclusão}

A detecção de maior velocidade na bola após o chute, para estudantes do sexo feminino, está ligada diretamente a assimetria de utilização e estímulo dado aos segmentos nas diferentes ações diárias e específicas do futsal. A maior participação em atividades esportivas de meninas que participam de escolinhas de futsal, pode também elevar a maturação biológica dentro da faixa etária cronológica analisada, e por outro lado, a baixa participação em atividades físicas específicas, retarda o amadurecimento e a qualificação motriz de meninas que apenas fazem atividades escolares.

Quanto às diferenças de velocidade entre os naipes masculino e feminino podem ser explicadas pela diferença hormonal e pelo início da puberdade, bem como o maior volume de treinamento de meninos que participam de escolinhas de futsal.

Sedo assim, é notório que meninas que apenas frequentem as aulas de educação física voltadas a prática do futsal, apresentam um volume de treino menor e consequentemente um déficit na velocidade da bola no chute, sendo inferior a meninas e meninos de mesma faixa etária e que praticam regularmente os treinamentos desta modalidade.

Diante dos achados, é possível determinar a importância da inserção de atividades técnicas bilaterais na prática do futsal ainda no processo de iniciação desportiva escolar, bem como a recomendação da participação em outras atividades que possam elevar sua capacidade física em pleno estágio de desenvolvimento, capacitando a versatilidade da aluna, 
proporcionando uma evolução constante.

O futsal feminino ainda enfrenta barreiras para a sua solidificação, a escola deve ser um gatilho para transpor tais obstáculos, criando uma geração de engajamento para esta causa e novas simpatizantes e praticantes desta modalidade, desenvolvendo assim um maior aporte de qualificação profissional e científico. Os autores sugerem novos estudos voltados ao público feminino no contexto do futsal, em específico ao nível escolar.

\section{Referências}

Andreis, G. M., Tannhauser, C. L., Biegelmeyer, U. h., Carmargo, M. E., Bernardi, F. C. \& Zanadrea, G. (2019). A inserção do público feminino no marketing esportivo. Revista Destaques Acadêmicos, 11 (2), 94-112.

Barbieri, F. A. (2005). Análise dos padrões cinemáticos do chute no futsal utilizando o membro dominante e o membro não dominante. (Monografia Trabalho de Conclusão de Curso Graduação em Educação Física) - Instituto de Biociências, Universidade Estadual Paulista, Rio Claro.

Barbieri, F. A., Gobbi, L. T. B., \& Lima Junior, R. S. (2006). Aspectos da corrida de aproximação entre o chute realizado com o membro dominante e não dominante. Revista Motricidade, 2 (2), 80-90.

Barbieri, F. A., Gobbi, L. T. B., Santiago, P. R. \& Cunha, S. A. (2010). Performance comparisons of the kicking of stationary and rolling balls in a futsal context. Sports Biomechanics, 9, 1-15. http://dx.doi.org/10.1080/14763141003690211

Barbieri, F. A., Santiago, P. R. P., Gobbi, L. T. B. \& Cunha, A. S. (2008). Análise cinemática da variabilidade do membro de suporte dominante e não dominante durante o chute no futsal. Revista Portuguesa de Ciência do Desporto, 8 (1), 68-76.

Brancher, E. A., Leite, G. E. \& Slomp, N. N. (2020). A origem dos gols em jogos de futsal feminino durante os jogos abertos de Santa Catarina 2019. Revista Brasileira de Futsal e Futebol, 12 (51), 705-712.

Culbert, K. M., Burt, S. A., Sisk, C., Nigg, J. T. \& Klump, K. L. (2014). The effects of circulating testosterone and pubertal maturation on risk for disordered eating symptoms in adolescent males. Psychological Medicine, 44 (11), 2271-2286. https://doi.org/10.1017/s0033291713003073

De Sousa, J., Alves, M. A. R. \& Pinheiro, M. H. N. P. (2020). Análise das ações ofensivas que resultaram em gols no quinto mundial universitário de futsal feminino de 2016. Revista Brasileira de Futsal e Futebol, 12 (49), 420-427. http://www.rbff.com.br/index.php/rbff/article/view/975

Endres, E., Belcaminho, G. C. T., Soares, B. H. (2003). Variação da velocidade da bola após o chute, entre categorias no futsal. PRONSAU-UNC. Universidade do Contestado. Concórdia-SC.

Freitas, D. L., Lausen, B., Maia, J. A., Lefevre, J., Gouveia, E. R., Thomis, M. \& Malina, R. M. (2015). Skeletal maturation, fundamental motor skills and motor coordination in children 7-10 years. Journal of Sports Science, 33 (9), 924-934. https://doi.org/10.1080/02640414.2014.977935

Gill, A. C. (2002). Como elaborar projetos de pesquisa. (4a ed.), Editora Atlas.

Godoi Filho, J. R. M. \& Farias, E. S. (2015). Aptidão física de escolares do sudoeste da Amazônia Ocidental em diferentes estágios de maturação sexual. Revista Brasileira de Educação Física e Esporte, 29 (4), 631-639. https://doi.org/10.1590/1807-55092015000400631

Guedes, D. P. (2011). Crescimento e desenvolvimento aplicado à educação física e ao esporte. Revista Brasileira de Educação Física e Esporte, 25 (supl), $127-140$.

Galatti, R. L. \& Paes, R. R. (2006). Fundamentos da pedagogia do esporte no cenário escolar. Movimento e Percepção, 6 (9), $16-25$.

Haywood, K. M. \& Getchell, N. (2004). Desenvolvimento Motor ao Longo da Vida. Artmed. p. 432.

Katis, A., Giannadakis, E., Kannas, T., Amiridis, I., Kellis, E. \& Lees, A. (2013). Mechanisms that influence accuracy of the soccer kick. Journal of Electromyography and Kinesiology. 23, 125-31. https://doi.org/10.1016/j.jelekin.2012.08.020

Malina, R. M. (2014). Top 10 research questions related to growth and maturation of relevance to physical activity, performance, and fitness. Research Quarterly for Exercise and Sport, 85 (2), 157-173. https://doi.org/10.1080/02701367.2014.897592

Marenucci, N. R., Oliveira, D. V., Freire, G. L. M., Nascimento Junior, J. R. A., Bennemann, R. M. \& Acencio, F. R. (2020). Ansiedade pré-competitiva, coesão de grupo e resiliência em atletas de futsal feminine. Revista Brasileira de Futsal e Futebol, 12 (48), $195-201$.

Martins, R.M. \& Ilha, F.R.S. (2020). The importance of futsal practice in the frequency and motivation of the students of the one special school. Research, Society and Development, 9 (8), 1-19. http://dx.doi.org/10.33448/rsd-v9i8.5540

Matsudo, V. K. R., Matsudo, S. M., Rezende, L. F. M. d. \& Raso, V. (2015). Handgrip strength as a predictor of physical fitness in children and adolescents. Revista Brasileira de Cineantropometria \& Desempenho Humano, 17 (1), 1-10. https://doi.org/10.5007/1980-0037.2015v17n1p1

Ishii, H., Yanagiya, T., Naito, H., Katamoto, S. \& Maruyama, T. (2009). Numerical study of ball behavior in side-foot soccer kick based on impact dynamic theory. Journal of Biomechanics, 42, 2712-20.

Nunome, H., Asai, T., Ikegami, Y \& Sakurai, S. (2002). Three-dimensional kinetic analysis of side-foot and instep soccer kicks. Medicine \& Science in Sports \& Exercise, 34 (12), 2028-2036. https://doi.org/10.1097/00005768-200212000-00025 
Research, Society and Development, v. 10, n. 10, e324101018977, 2021

(CC BY 4.0) | ISSN 2525-3409 | DOI: http://dx.doi.org/10.33448/rsd-v10i10.18977

Nunome, H., Ikegami, Y., Kozakai, R., Apriantono, T. \& Sano, S. (2006). Impact phase kinematics of instep kicking soccer. Journal of sports sciences, 24 (1), $11-22$.

Rebelo, A., Brito, J., Maia, J., Coelho-e-Silva, M. \& Seabra, A. (2013). Anthropometric characteristics, physical fitness and technical performance of under-19 soccer players by competitive level and field position. International Journal of Sports Medicine, 34 (4), 312-317. https://doi.org/10.1055/s-0032-1323729

Reis, F. P. G. \& Arruda, I. E. A. (2011). Uma história do futebol feminino brasileiro: superando preconceitos. Revista Digital EFDeportes, 16 (163), 1. https://www.efdeportes.com/efd163/uma-historia-do-futebol-feminino-brasileiro.htm

Rother, R. \& Scalco, A. (2020). Análise da origem dos gols de uma equipe de futsal marcados em jogos como visitante e mandante. Revista Brasileira de Futsal e Futebol, 12 (51), 720-725.

Santiago, P. R. P. (2006). Comparação do padrão cinemático do chute no futebol entre jogadores de dois países. (Mestrado em Motricidade Humana) Instituto de Biociências, Universidade Estadual Paulista, Rio Claro.

Santiago, P. R. P., Moura, F. A., Barbieri, F. A., Lima Júnior, R. S. \& Cunha, S. A. Relação do padrão cinemático de movimento com desempenho do chute em cobranças de falta no futebol. Revista Brasileira de Biomecânica, 8, 15-60.

Santos, M. M., Ferreira, A. H. S., Costa, M. C., Guimarães, F. J. S. \& Ritti-Dias, R. M. (2011). Contribuição da massa muscular na força de preensão manual em diferentes estágios maturacionais. ConScientiae Saúde, 10 (3), 487-493.

Slimani, M., Bragazzi, N. L., Tod, D., Dellal, A., Hue, O. \& Cheour, F. (2016). Do cognitive training strategies improve motor and positive psychological skills development in soccer players? Insights from a systematic review. Journal of Sports Sciences, 34, $2338-2349$. https://doi.org/10.1080/02640414.2016.1254809

Silva, M. V. S., Caríssimo, J. M. N., Silva, C. M., \& Silva, S. A. (2020). Análise da incidência de gols e suas características nas fases finais da liga nacional de futsal do Brasil de 2019. Revista Brasileira de Futsal e Futebol, 12 (51), 765-771. http://www.rbff.com.br/index.php/rbff/article/view/1049

Teixeira, F. G. (2004). Comparações dos padrões cinemáticos de chute realizados por participantes de 15 e 17 anos. (Mestrado em Motricidade Humana) Instituto de Biociências, Universidade Estadual Paulista, Rio Claro.

Voser, R. C. (2019). Futsal: princípios técnicos e táticos. Editora da Ulbra.

Zanatta, A. M., Soares, B., \& Pasqualotti, A. (2020). Velocidade da bola após o chute, nas categorias de base no futsal feminino. Revista Brasileira de Futsal e Futebol, 12 (48), 187-194. 